Article

\title{
Dynamical Response of Particles in Asymmetric Ratchet Potential
}

\author{
Usman A. Marte ${ }^{1, *}$, Uchechukwu E. Vincent ${ }^{2,3, \dagger, *}$, Abdulahi N. Njah ${ }^{4,5, \dagger}$ and \\ Biodun S. Badmus $4, \dagger$
}

1 Department of Mathematics and Statistics, University of Maiduguri, P.M.B. 1069 Maiduguri, Nigeria

2 Department of Physics, Lancaster University of Lancaster, Lancaster LA1 4YB, UK

3 Department of Physical Sciences, Redeemer's University, P.M.B. 3005 Redemption City, Nigeria

4 Department of Physics, Federal University of Agriculture, P.M.B. 2240 Abeokuta, Nigeria;

E-Mails: njahabdul@yahoo.com (A.N.N.); badmus@physics.unaab.edu.ng (B.S.B.)

5 Department of Physics, University of Lagos, P.M.B. 56 Akoka-Yaba, Lagos, Nigeria

$\dagger$ These authors contributed equally to this work.

* Authors to whom correspondence should be addressed; E-Mails: auamarte@yahoo.com (U.A.M.); ue_vincent@yahoo.com or u.vincent@lancaster.ac.uk (U.E.V.);

Tel.: +234-802-357-6675 (ext. 123) (U.A.M.).

External Editor: Soren Toxvaerd

Received: 28 July 2014; in revised form: 23 September 2014 / Accepted: 15 October 2014 /

Published: 27 October 2014

\begin{abstract}
The dynamics and response of particles in an asymmetric potential of the ratchet type is considered. An approximate asymmetric ratchet potential, which allows us to apply an analytical approach to investigate the systems' response, is proposed based on the power series interpolation scheme. The method of multiple scales (MMS) is then employed to obtain an approximate solution at the principal resonance. Comparison between results obtained from the approximated and exact potentials show excellent agreement. The frequency response curves (FRCs) for different forcing are presented. The numerically computed FRCs were compared with the theoretical result to show the extent of agreement. Furthermore, by using perturbation methods, the stable and unstable domains of the solution were obtained.
\end{abstract}

Keywords: ratchets; asymmetry potential; resonance; multiple time scale; chaos 


\section{Introduction}

In recent times, there has been increasing interest in the study of the transport properties of nonlinear systems that extract usable work from unbiased non-equilibrium fluctuations [1]; one of the reasons being that non-equilibrium dynamical systems are potential models for describing a wide range of natural and mechanical systems [2]. For instance, rectifying the motion of the Josephson phase in superconducting quantum interference devices (SQUIDs) or long Josephson junctions, vortices in superconductors and Josephson junction arrays are some physical promising implementations of a ratchet device (see [3] and the references therein).

In general, a ratchet can be modelled, for instance, by considering a Brownian particle in a periodic asymmetric potential, which is acted upon by an external time-dependent force of zero average. The basic concept is governed by the second law of thermodynamics, wherein noise-induced directed transport in a spatially periodic system in thermal equilibrium is ruled out [4,5]. Therefore, to generate transport, Brownian motors or ratchet devices could be used for modelling such systems. Interestingly, deterministically-induced chaos could in many instances mimic the role of noise, thereby steering the system to exhibit current flow in either direction. Within this framework, diverse research works, ranging from chaotic transport and transport control via nonlinear control theory, to control by synchronization, have been done [5-25].

Despite the huge research progress made in the study of ratchet systems to date, only a few recent works reported the response of particles in asymmetric ratchet potential. For instance, Nana-Nbendjo et al. [24] carried out an analytical study of its stability, examined resonance oscillations for two indirectly-coupled ratchets and reported the existence of multi-resonance. In a very recent and related work, Vincent et al. [25] considered the collective dynamics of a network of particles in a ratchet potential and observed collective resonance. Remarkably, these works were mainly concerned with numerical results, with no recourse to the analytical treatment of the frequency response.

In this paper, we propose an approach that allows us to obtain, analytically and numerically, the dynamical response of a deterministic (i.e., noiseless) inertia ratchet system by means of a polynomial interpolation scheme. Our motivations for investigating deterministic ratchets transporting massive particles is derived from the strong inertial effects leading to current reversals, as well as their natural capability for developing chaotic dynamics; all of which, in nanotechnology, makes it a practical, viable alternative for the purpose of rectification (see [3] and the references therein). Indeed, it was pointed out that the inertia system can perform more effectively and efficiently than the more popular over-damped Brownian motors originally inspired by cellular biology [3]. On the other hand, the need for polynomial interpolation has been informed by the need to re-express the ratchet potential containing the sine functions in a form that will be readily accessible to analytical treatment using the method of multiple timescales (MMS). Application of the MMS involves the composition of an exponential function of these sine functions, which makes the solvability conditions difficult to obtain. Thus, the problem was easily overcome by means of the polynomial approximation. With our proposed approach, we obtain an approximate asymmetric ratchet potential, which allows us to apply the method of multiple scales in order to obtain the frequency response analytically and then confirm the result by direct numerical solution of the nonlinear base equation. 
The rest of the paper is organized as follows. In the next section, we give a brief description of the inertia ratchet model. Section 3 describes our approximation technique, an analysis using the method of multiple scales, as well as the examination of the stability of the steady state of the attractors. The paper is concluded in Section 4.

\section{The Inertia Ratchet}

Consider the one-dimensional equation of motion of a particle driven by a periodic time-dependent external force under the influence of an asymmetric potential of the ratchet type. The time average of the external deterministic force is zero, and the dimensionless equation of motion is given by [5-25]:

$$
\ddot{x}+b_{1} \dot{x}+\frac{d V(x)}{d x}=F_{0} \cos (\omega t)
$$

where $b_{1}$ is a dimensionless friction coefficient, $F_{0}$ is the amplitude of the external forcing, $\omega$ is the external driving frequency and $V(x)$ is an external asymmetric periodic potential of the ratchet type given by [6] as:

$$
V(x)=c-\frac{1}{4 \pi^{2} \delta}\left[\sin \left(2 \pi\left(x-x_{0}\right)\right)+0.25 \sin \left(4 \pi\left(x-x_{0}\right)\right)\right]
$$

where the potential is shifted by $x_{0}$ in order to make the minima of the potential to be located at integer values. The parameters $\delta=1.61432$ and $c=0.0173$. System (1) has been extensively studied in both the low [26] and moderate [9,11-13] damping regimes, where chaotic, as well as regular dynamics could be found; while in [27,28], the effects of noise were investigated. In order to facilitate the analysis using the method of multiple scales, Equation (1) may be written as:

$$
\ddot{x}+\omega_{0}^{2} x=-b_{1} \dot{x}+\frac{1}{4 \pi \delta}\left[2 \cos \left(2 \pi\left(x-x_{0}\right)\right)+\cos \left(4 \pi\left(x-x_{0}\right)\right)\right]+\omega_{0}^{2} x+F_{0} \cos \omega t
$$

where $\omega_{0}$ is the natural frequency of the system.

\section{Results and Discussion}

\subsection{Approximation Technique}

Our goal is to seek for an approximate asymptotic solutions of Equation (3) using multiple scales $[29,30]$. In order to achieve this, we first obtain an approximation to the potential given by Equation (2) by considering a set of $N+1$ data points, from which we fit an $N$-th degree polynomial that passes through these points by means of the Lagrange interpolating functions. Regardless of how the $N$-th degree polynomial is constructed, the resulting polynomial would be the same. Now, let $g(x)$ be the exact function, with known $N+1$ discrete values used to establish an interpolating polynomial or approximating function $f(x)$. The function $f(x)$ should pass through all specified $N+1$ points, also referred to as data points or nodes. For a given set of interpolating points $\left(x_{i}, g\left(x_{i}\right)=g_{i}, i=0, \ldots, N\right)$, there exists only one polynomial that passes through a given set of $N+1$ points. Its form, expressed in power series, is given by:

$$
f(x)=\sum_{i=0}^{N} a_{i} x^{i}
$$


where $a_{i}(i=0,1,2, \ldots, N+1)$ are unknown coefficients. In principle, $f(x)$ must match $g(x)$ at the selected data points, such that:

$$
\left(x_{j}: f\left(x_{j}\right)=g_{j}=\sum_{k=0}^{N} a_{j} x_{j}^{k},\{j=1, \ldots, N\}\right)
$$

From the above matrix, the unknown coefficients $a_{i}$ can be obtained. The second term on the right-hand side of Equation (3) represents $\mathrm{d} V / \mathrm{d} x$. To obtain the approximation, we choose evenly-spaced data points and evaluate the coefficients $a_{i}$ from Equation (5).

We begin by dividing the interval $[0,1]$ into two equal sub-intervals, so that the interpolating polynomial becomes $f(x)=a_{0}+a_{1} x+a_{2} x^{2}$, where $a_{0}=-0.0105557, a_{1}=0.335818, a_{2}=-0.335818$. Next, we consider four sub-intervals and obtain the interpolating polynomial as $f(x)=a_{0}+a_{1} x+a_{2} x^{2}+a_{3} x^{3}+a_{4} x^{4}$, where $a_{0}=-0.0105557, a_{1}=-0.501061, a_{2}=5.75165, a_{3}=-12.4042, a_{4}=7.15365$. For five sub-intervals, the interpolating polynomial becomes $f(x)=a_{0}+a_{1} x+a_{2} x^{2}+a_{3} x^{3}+a_{4} x^{4}+a_{5} x^{5}$, where $a_{0}=-0.0105557$, $a_{1}=1.69548, a_{2}=-11.7413, a_{3}=34.1655, a_{4}=-43.5321, a_{5}=19.4124$.

Finally, for ten sub-intervals, we obtain the interpolating polynomial:

$$
f(x)=a_{0}+a_{1} x+a_{2} x^{2}+a_{3} x^{3}+a_{4} x^{4}+a_{5} x^{5}+a_{6} x^{6}+a_{7} x^{7}+a_{8} x^{8}+a_{9} x^{9}+a_{10} x^{10}
$$

From Equation (5), the following values of $a_{i}$ were obtained: $a_{0}=-0.0105557, a_{1}=1.31333$, $a_{2}=-9.63468, a_{3}=77.7829, a_{4}=-571.273, a_{5}=2424.3, a_{6}=-5748.15, a_{7}=7839.35, a_{8}=-6123.42$, $a_{9}=2547.15$ and $a_{10}=-437.428$. With further division of the interval to twenty equal sub-intervals, the interpolating polynomial becomes "drowned" in its rounding errors. Remarkably, when the component of $V(x)$ with a half period is set to zero, the dynamics of System (1) could simply be likened to a model of a superconducting Josephson junction considered by Huberman et al. [31]. However, in this limit, and for a $2 \pi$ periodic potential function, as in [31], the best polynomial fit can be obtained using eighth-order polynomial by dividing the $2 \pi$ periodic function into eight equal parts.

In the following analysis, we choose the case of ten sub-intervals, which gives an excellent agreement, and a prime period of the system is considered, which can be extended to infinitum by the use of the $\operatorname{Mod}[x, 1]$ in the interpolating polynomial obtained in Equation (6). To validate the above result, we first compare the approximated $\mathrm{d} V / \mathrm{d} x$ with the results obtained by direct numerical simulation, as shown in Figure 1, where we superpose the plots for the exact (red lines) and the approximation (green lines) obtained using Equation (6) in the interval $0 \leq x \leq 1$. The interpolating polynomial can be extended to infinitum by the use of the Mod function. Clearly, one can see an excellent qualitative agreement between the two plots.

First, we computed the chaotic attractor using Equation (1) with the exact potential (2) and compare with the attractor obtained using potential (6). Clearly, as shown in Figure 2, the two attractors (exact in green and approximated in read) are qualitatively in good agreement, retaining the essential features, including the shapes, basins and boundedness of the attractor. 
Figure 1. Comparison of exact $g(x)=\mathrm{d} V / \mathrm{d} x$ (red) and the approximate $f(x)=\mathrm{d} V / \mathrm{d} x$ (Green) in the interval [0,1]; (a) division into two sub-intervals; (b) division into four sub-intervals; (c) division into five sub-intervals; (d) division into 10 sub-intervals.

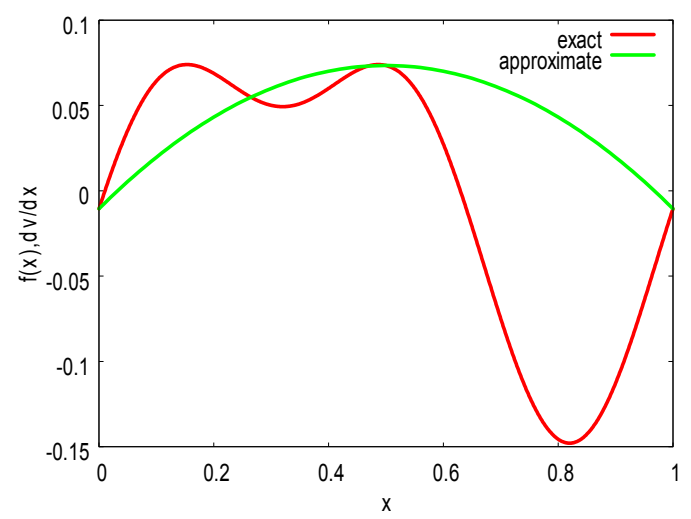

(a)

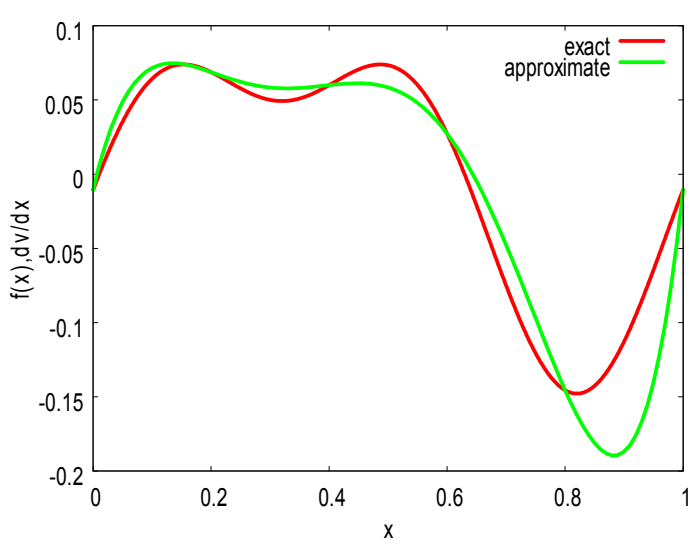

(c)

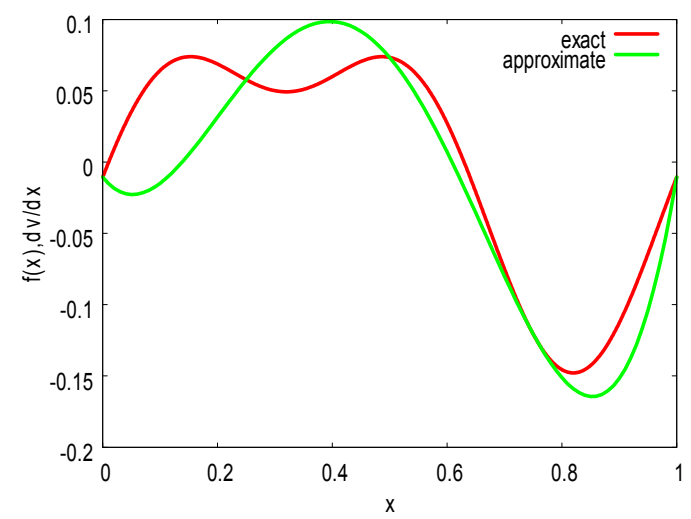

(b)

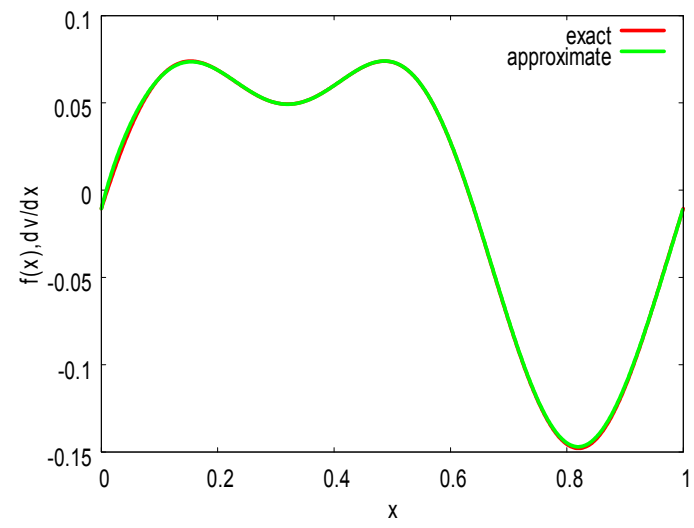

(d)

Figure 2. Comparison between the attractors obtained from direct numerical simulation and the approximated value. The parameters are: $b_{1}=0.1, F_{0}=0.08, \omega=0.67, x_{0}=-0.19$, $\delta=1.61432$ and $c=0.0173$. Direct Numerical in green and approximated in read.

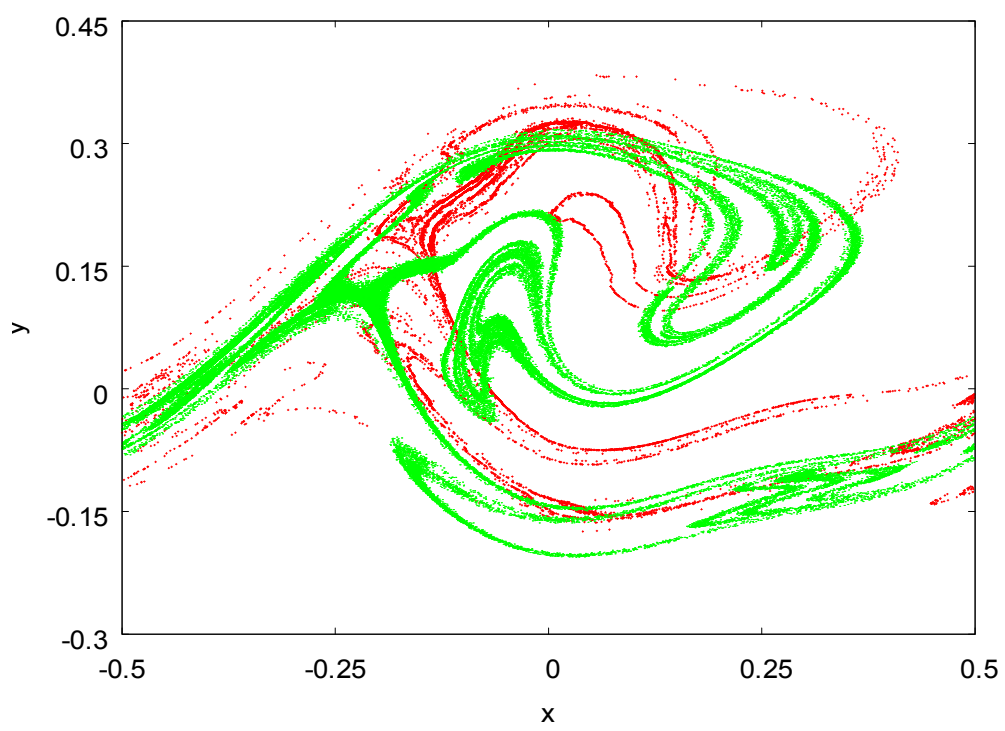




\subsection{Analysis with the Method of Multiple Scales}

Now, considering Equations (1) and (6), one seeks for asymptotic solutions by employing the method of multiple scales [26,32]. For a small, but finite, value of $x$, one considers a power series solution of the form:

$$
x(t: \varepsilon)=x_{0}\left(T_{0}, T_{1}\right)+\varepsilon x_{1}\left(T_{0}, T_{1}\right)+\ldots
$$

where $T_{0}=t \mathrm{a}$ is a fast scale and $T_{1}=\varepsilon t$ is a slow scale. The first and second derivatives are given by:

$$
\frac{d}{d t}=D_{0}+\varepsilon D_{1}+\ldots, \quad \frac{d^{2}}{d t^{2}}=D_{0}^{2}+2 \varepsilon D_{0} D_{1}
$$

Equating the coefficients of equal powers of $\varepsilon$ results in $\mathrm{O}\left(\varepsilon^{0}\right)$ and $\mathrm{O}\left(\varepsilon^{1}\right)$, we obtain respectively,

$$
D_{0}^{2} x_{0}+\omega_{0}^{2} x_{0}=0
$$

and

$$
D_{0}^{2} x_{1}+\omega_{0}^{2} x_{1}=-2 D_{0} D_{1} x_{0}-b D_{0} x_{0}+F_{0} \cos (\omega t)+\alpha f\left(x_{0}\right)+\omega_{0}^{2} x_{0}
$$

The general solution of Equation (10) can be written in the form:

$$
x_{0}=A\left(T_{1}\right) e^{i \omega_{0} T_{0}}+\bar{A}\left(T_{1}\right) e^{-i \omega_{0} T_{0}}
$$

where $\alpha=\varepsilon \alpha_{10}, \omega_{0}=\varepsilon \omega_{10}, b=\varepsilon b_{1} ; \omega_{10}$ and $\alpha_{10}$ are the natural frequency and scaling factor, respectively.

The complex conjugate pair quantities $A\left(T_{1}\right)$ and $\bar{A}\left(T_{1}\right)$ are arbitrary functions, to this order of approximation, and are determined by imposing the solvability conditions in the next order of approximation. Substituting Equation (11) into Equation (10) results in:

$$
\begin{aligned}
D_{0}^{2} x_{1}+\omega_{0}^{2} x_{1}= & \left(-i \omega_{0}\left(2 A^{\prime}-b A\right)+\alpha J_{1}(A)+\omega_{0}^{2}\right) e^{i \omega_{0} T_{0}}+\alpha\left(J_{2}(A) e^{2 i \omega_{0} T_{0}}\right. \\
& +J_{3}(A) e^{3 i \omega_{0} T_{0}}+J_{4}(A) e^{4 i \omega_{0} T_{0}}+J_{5}(A) e^{5 i \omega_{0} T_{0}}+J_{6}(A) e^{6 i \omega_{0} T_{0}} \\
& \left.J_{7}(A) e^{7 i \omega_{0} T_{0}}+J_{8}(A) e^{8 \omega_{0} T_{0}}+J_{9}(A) e^{9 i \omega_{0} T_{0}}+J_{10}(A) e^{10 i \omega_{0} T_{0}}+J_{11}(A)\right)+\frac{1}{2} F_{0} e^{i \omega T_{0}}
\end{aligned}
$$

The quantities $J_{i}(A), i=1,2, \ldots, 12$ are defined in the Appendix.

\subsection{Principal Resonance}

Here, we analyse the case where $\omega_{0}$ is close to $\omega$; where we set $\omega=\omega_{0}+\varepsilon \sigma$ and $\sigma$ is the detuning parameter. For a bounded solution, one has to impose a condition to eliminate secular terms in Equation (12). This requires that:

$$
D_{0}^{2} x_{1}+w_{0}^{2} x_{1}=\left(-i w_{0}\left(2 A^{\prime}-b_{1} A\right)+w_{0}^{2} A+J_{1}(A)\right) e^{i w_{0} T_{0}}+[H . O . T]+\frac{1}{2} F_{0} e^{i w T_{0}}
$$

where [H.O.T] refer to higher order terms in $e^{i w_{0} T_{0}}$. To solve Equation (13), $A$ is written in polar form as:

$$
A\left(T_{1}\right)=\frac{1}{2} a e^{i \phi}
$$

where $a$ and $\phi$ are real functions. Substituting Equation (14) in Equation (13) results in:

$$
-i \omega_{0}\left(a^{\prime}+i a \phi^{\prime}\right)-0.5 i \omega_{0} b_{1} a+\alpha s(a)+\omega_{0}^{2} a+\frac{F_{0}}{2} e^{i\left(\sigma T_{1}-\phi\right)}
$$

where $s(a)=0.65667 a+29.16859 a^{3}+757.59375 a^{5}+2143.57227 a^{7}+626.8377 a^{9}$. 
Separating real and imaginary parts results in the following Equations:

$$
\begin{aligned}
& a^{\prime}=-\frac{b_{1}}{2} a+\frac{F_{0}}{2 \omega_{0}} \sin \theta \\
& a \theta^{\prime}=a \sigma+\frac{\alpha}{2 \omega_{0}} s(a)+\frac{\omega_{0}}{2} a+\frac{F_{0}}{2 \omega_{0}} \cos \theta
\end{aligned}
$$

where $\theta=\sigma T_{1}-\phi$. The stability of the solution can be examined by locating the singular points and their neighbourhoods. When the amplitude and the phase do not change at the singular points, the response is said to be a steady-state motion. From Equation (16), the position and velocity of the particle can be found at any point in time, thus enabling the knowledge of the particles' current at any point in time.

The steady-state motion occurs when $a^{\prime}=\theta^{\prime}=0$ and $\phi^{\prime}=\sigma$, which corresponds to the singular points of Equation (16), which, in turn, correspond to the solutions of:

$$
\begin{aligned}
& \frac{1}{2} b_{1} a=\frac{F_{0}}{2 \omega_{0}} \sin \theta \\
& a \sigma+\frac{\alpha}{2 \omega_{0}} S(a)+\frac{\omega_{0}}{2} a=-\frac{F_{0}}{2 \omega_{0}} \cos \theta
\end{aligned}
$$

By squaring and adding the Equations in (17), we obtain the expression for the frequency response as:

$$
\omega_{0}^{2} b_{1}^{2} a^{2}+4 \omega_{0}^{2}\left[\sigma a+\frac{\alpha}{\omega_{0}} s(a)+\omega_{0} a\right]^{2}=F_{0}^{2}
$$

Equation (18) is an implicit equation for the amplitude of the response as a function of the detuning parameter $\sigma$ (frequency of the excitation) and the amplitude of the excitation, which is called the frequency response. By fixing the system parameters as $b_{1}=0.1, \omega_{0}=1.0, \alpha=0.01$, we show a plot of the frequency response curves for different values of the external excitation amplitude, $F_{0}=0.03,0.06$ and 0.12 . The theoretical and the numerical response amplitude are first compared from Equations (16) and (18). Again, we find excellent agreement, as shown in Figure 3.

Figure 3. Response amplitude theoretical (red) and numerical (green) for (a) $F_{0}=0.12$, (b) $F_{0}=0.06$ and (c) $F_{0}=0.03$, showing a very good agreement in the two results.

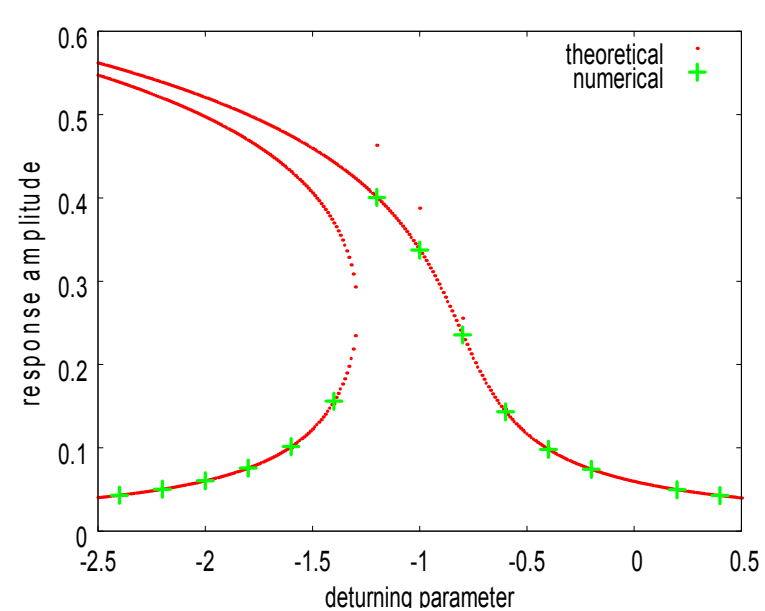

(a)

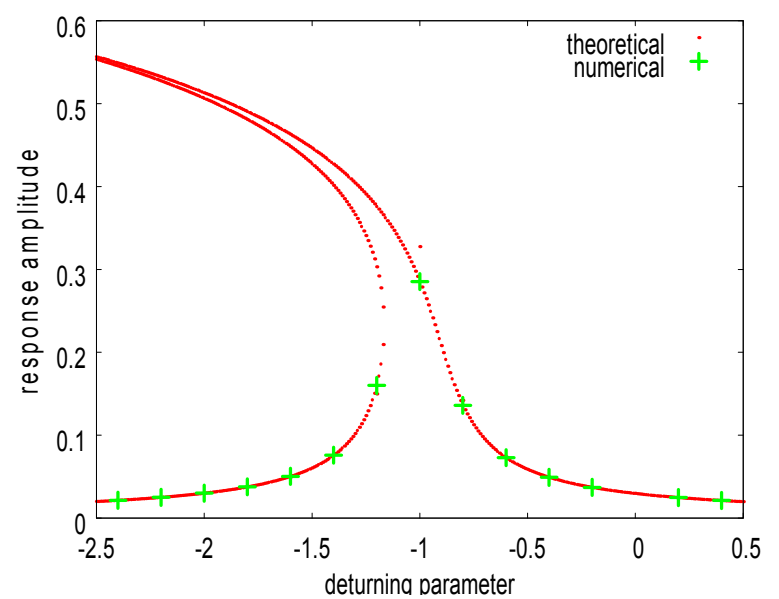

(b) 
Figure 3. Cont.

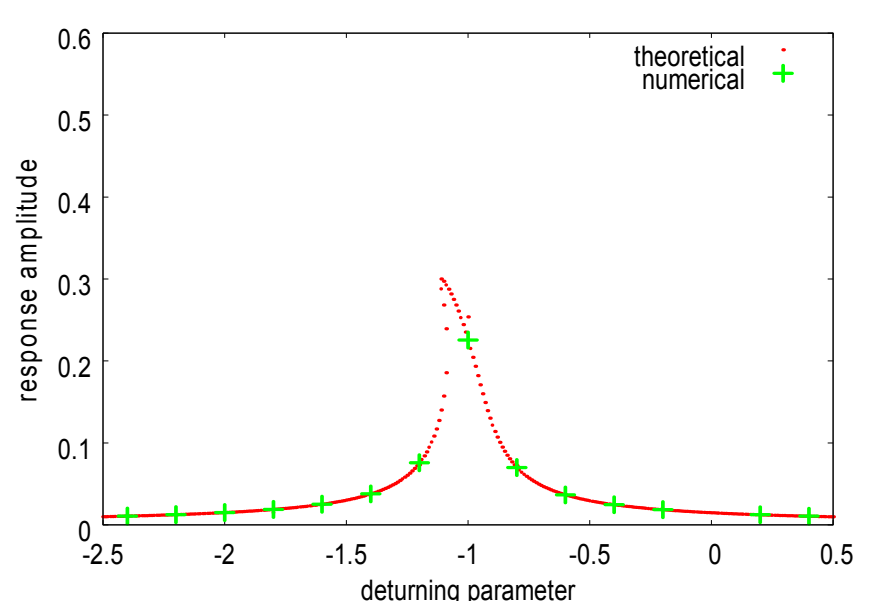

(c)

By comparing the frequency response curves for different values of the external excitation amplitude, $F_{0}=0.03,0.06$ and 0.12 , we observe, clearly, a jump phenomenon for some values of the forcing amplitude $F_{0}$. As shown in Figure 4, the jump phenomenon is not visible at $F_{0}=0.03$, but at $F_{0}=0.06$. At higher values of $F_{0}$, the jump is clearly visible. Notably, as $F_{0}$ increases, the point at which the jump occurs moves away from the resonance point (i.e., at $\sigma=-1$ ), as shown in Figure 3. The occurrence of the jump phenomenon in the frequency resonance curve is evidence of the system's strong dependency on the initial conditions. Strong dependence on the initial conditions is typical of deterministic underdamped ratchets [26].

Figure 4. Frequency response as a function of the detuning parameter, $\sigma$, for different values of $F_{0}=0.03,0.06$ and 0.12 , as indicated.

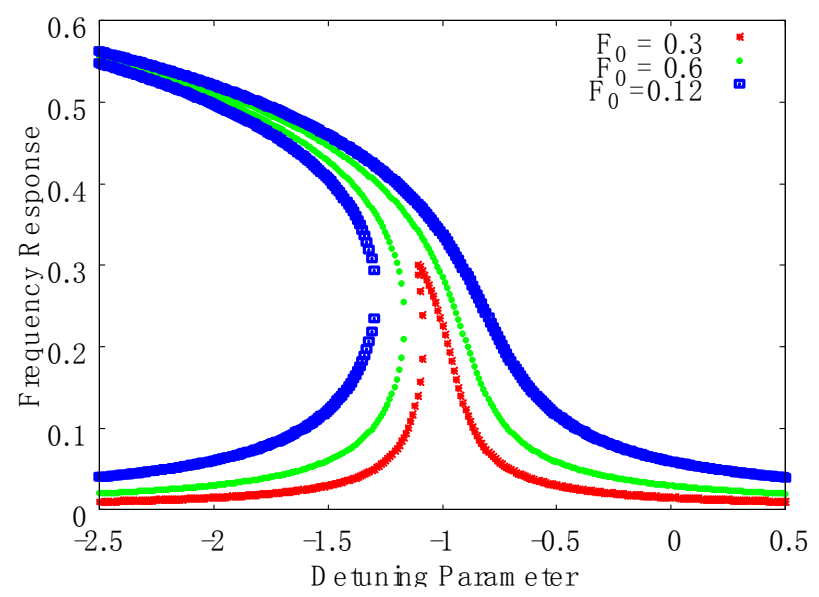

\subsection{Stability of the Steady-State Solution}

The stability of the steady-state motion can be examined from Equation (17) by introducing a small perturbation of the form:

$$
\begin{aligned}
& a=a_{0}+a_{1} \\
& \theta=\theta_{0}+\theta_{1}
\end{aligned}
$$


Substituting Equation (19) in Equation (17) and keeping only the linear terms in $a_{1}$ and $\theta_{1}$, noting that $a_{0}$ and $\theta_{0}$ satisfy Equation (18), results in the following:

$$
\begin{aligned}
& a_{1}^{\prime}=-\frac{b_{1}}{2} a_{1}+\left[\frac{F_{0}}{2 \omega_{0}} \cos \theta_{0}\right] \theta_{1} \\
& \theta_{1}^{\prime}=\left[\frac{\alpha}{a_{0}^{2} \omega_{0}}\left(u\left(a_{0}\right)-s\left(a_{0}\right)\right)-\frac{F_{0}}{2 a_{0} \omega_{0}} \cos \theta_{0}\right] a_{1}-\left[\frac{F_{0}}{2 \omega_{0} a_{0}} \sin \theta_{0}\right] \theta_{1}
\end{aligned}
$$

where $u(a)=\frac{1}{2} a_{1} a+3 \frac{3}{8} a_{3} a^{3}+5 \frac{10}{32} a_{5} a^{5}+7 \frac{35}{128} a_{7} a^{7}+9 \frac{126}{512} a_{9} a^{9}$.

Thus, the stability of the steady-state motion depends on the eigenvalues of the coefficient matrix of the right-hand side of Equation (21), given by the equation:

$$
\left|\begin{array}{cc}
-\frac{b_{1}}{2}-\lambda & -\frac{1}{\omega_{0}}\left(a_{0} \sigma+\alpha s\left(a_{0}\right)+\omega_{0}^{2} a_{0}\right) \\
\alpha u\left(a_{0}\right)+\frac{1}{\omega_{0} a_{0}^{2}}\left(a_{0} \sigma+\alpha s\left(a_{0}\right)+\omega_{0}^{2} a_{0}\right) & -\frac{b_{1}}{2}-\lambda
\end{array}\right|=0
$$

Choose the values $\omega_{0}=1.0, \alpha=0.01$ and $b_{1}=0.1$. In order to obtain the stability conditions, we employ the Routh-Hurwitz criterion. Given a polynomial:

$$
P(\lambda)=\lambda^{n}+a_{1} \lambda^{n-1}+\ldots+a_{n-1} \lambda+a_{n}
$$

where the coefficients $a_{i}, i=1,2,3, \ldots n$, are real constants. Define as Hurwitz the $n$ matrices using the coefficients $a_{i}$ of the characteristics polynomial:

$$
\begin{gathered}
H_{1}\left(a_{1}\right)=\left(a_{1}\right), H_{2}=\left(\begin{array}{cc}
a_{1} & 1 \\
a_{3} & a_{2}
\end{array}\right), H_{3}=\left(\begin{array}{ccc}
a_{1} & 1 & 0 \\
a_{3} & a_{2} & a_{1} \\
a_{5} & a_{4} & a_{3}
\end{array}\right) \text { and } \\
H_{n}=\left(\begin{array}{cccccc}
a_{1} & 1 & 0 & 0 & \ldots & 0 \\
a_{3} & a_{2} & a_{1} & 1 & \ldots & 0 \\
a_{5} & a_{4} & a_{3} & a_{2} & \ldots & 0 \\
\vdots & \vdots & \vdots & \vdots & \ldots & \vdots \\
0 & 0 & 0 & 0 & \ldots & a_{n}
\end{array}\right)
\end{gathered}
$$

where $a_{i}=0$ if $j>n$. All of the roots of the polynomials $P(\lambda)$ are negative or have a negative real part if and only if the determinants of all of the Hurwitz matrices are positive: det. $H_{j}>0, j=1,2, \ldots, n$. When $n=2$, the Routh-Hurwitz criterion simply becomes det. $\mathrm{H}_{1}=a_{1}>0$ and det. $\mathrm{H}_{2}=\operatorname{det}\left(\begin{array}{cc}a_{1} & 1 \\ a_{3} & a_{2}\end{array}\right)=a_{1} a_{2}>0$ or $a_{1}>0$ and $a_{2}>0$ and $a_{3}=0$ by definition. Applying the above criteria to our system Equation (21) results in the stability condition given by:

$$
\frac{b_{1}^{2}}{4}+\left(\alpha u\left(a_{0}\right)+\frac{1}{a_{0}^{2}}\left(a_{0} \sigma+\alpha s\left(a_{0}\right)+\omega_{0}^{2} a_{0}\right)\right)\left(a_{0} \sigma+\alpha s\left(a_{0}\right)+\omega_{0}^{2} a_{0}\right)>0
$$

By choosing the following parameter values, $\omega_{0}=1.0, \alpha=0.01$ and $b_{1}=0.1$, we show in Figure 5 the variation of the stability boundaries with increasing nonlinearity $\alpha$. The stability analysis and the numerical solutions (green points) in Figure 3 show clearly the realizable values of the amplitudes where abrupt changes in a very small window of the detuning parameter can take place. This relates to the result reported in [26], where a sudden jump in the current in a very narrow rectification amplitude window was reported. 
Figure 5. Boundary separating stable and unstable regions for different values of $\alpha$. (a) $\alpha=0.01$; (b) $\alpha=0.05$; and (c) $\alpha=0.1$.

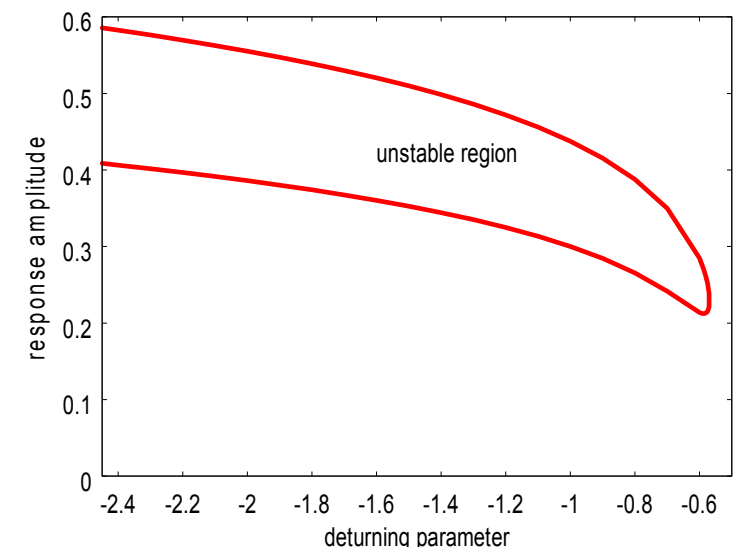

(a)

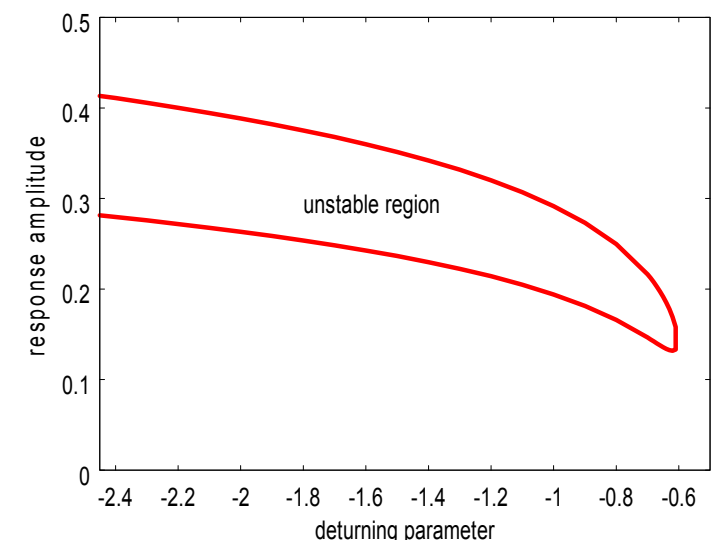

(b)

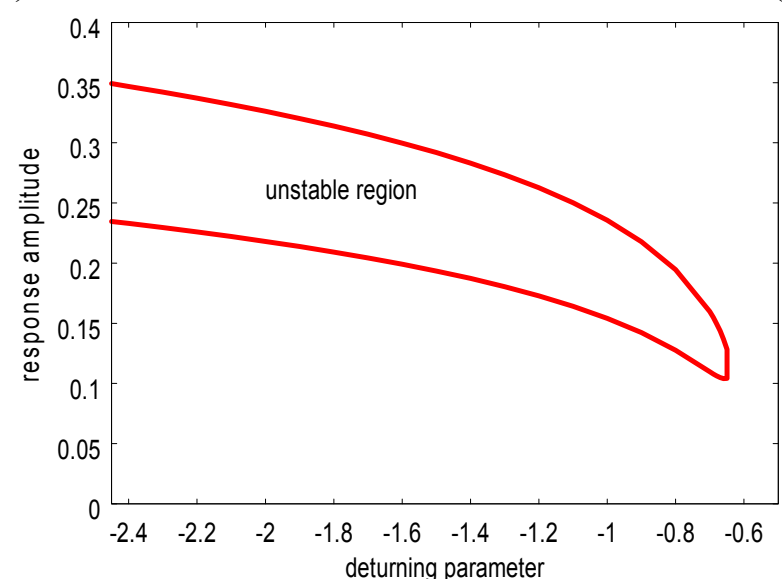

(c)

\section{Conclusions}

In this paper, we studied the dynamics of a deterministic inertia ratchet system subjected to an external periodic forcing. This system is extremely sensitive to initial conditions and has a natural capability for exhibiting chaotic dynamics, which in practice makes it a suitable model in nanotechnology for the purpose of rectification [3]. Due to this strong nonlinear property, it can perform more effectively and efficiently than the popular over-damped models that find useful applications in cellular biology [3]. Here, we have obtained the frequency response analytically by approximating the ratchet potential with a power series by means of an interpolation scheme. The method of multiple scales was used to obtain an analytic solution at the primary resonance. The frequency response shows evidence of sensitivity to initial conditions manifesting as jump phenomena at different amplitudes of the external forcing. Numerical solutions of the response amplitude were computed and compared with the theoretical results. The stability of the solution was analysed by perturbation methods to obtain the boundary separating the stable and the unstable regions using the Routh-Hurwitz criterion. The results showed that as the nonlinearity increases, the region of stability decreases. The realizable values of the amplitudes where abrupt changes in a very small window of the detuning parameter can take place were found. This is 
similar to the result reported in [26], where a sudden jump in the current in a very narrow rectification amplitude window was reported.

\section{Acknowledgments}

Uchechukwu E. Vincent is supported by the Royal Society of London through their Newton International Fellowship Alumni scheme. We acknowledge and thank all of the reviewers for their constructive and critical comments that were very useful for improving the quality of this paper.

\section{Author Contributions}

Uchechukwu E. Vincent proposed the work. Usman A. Marte carried out all of the mathematical analysis under the joint supervision of Uchechukwu E. Vincent and Abdulahi N. Njah The code for Figure 2 was written by Uchechukwu E. Vincent. Manuscript preparation, presentation and editing were done by all of the authors.

\section{Appendix}

$$
\begin{aligned}
& J_{1}(A)=-0.0106+\left(1.3133 A+233.349 A^{2} \bar{A}+24243 A^{3} \bar{A}^{2}+274377 A^{4} \bar{A}^{3}+320941 A^{5} \bar{A}^{4}\right) \\
& J_{11}(A)=-\left(19.2694 A \bar{A}+3427.64 A^{2} \bar{A}^{2}+114963 A^{3} \bar{A}^{3}+428639 A^{4} \bar{A}^{4}+110232 A^{5} \bar{A}^{5}\right) \\
& J_{2}(A)=-\left(9.6347 A^{2}+2285.09 A^{3} \bar{A}+86222.3 A^{4} \bar{A}^{2}+342912 . A^{5} \bar{A}^{3}+91859.8 A^{6} \bar{A}^{4}\right) \\
& J_{3}(A)=\left(77.7829 A^{3}+12121.5 A^{4} \bar{A}+164626 A^{5} \bar{A}^{2}+213961 A^{6} \bar{A}^{3}\right) \\
& J_{4}(A)=-\left(571.273 A^{4}+34488 A^{5} \bar{A}+171456 A^{6} \bar{A}^{2}+52491.4 A^{7} \bar{A}^{3}\right) \\
& J_{5}(A)=\left(2424.3 A^{5}+54875.5 A^{6} \bar{A}+91697.4 A^{7} \bar{A}^{2}\right) \\
& J_{6}(A)=-\left(5748.15 A^{6}+48987.4 A^{7} \bar{A}+19684.3 A^{8} \bar{A}^{2}\right) \\
& J_{7}(A)=\left(7839 A^{7}+22924.4 A^{8} \bar{A}\right) \\
& J_{8}(A)=-\left(6123.42 A^{8}+4374.28 A^{9} \bar{A}\right) \\
& J_{9}(A)=2547.15 A^{9} \\
& J_{10}(A)=-437.428 A^{10}
\end{aligned}
$$

\section{Conflicts of Interest}

The authors declare no conflict of interest.

\section{References}

1. Hanggi, P.; Marchesoni, F. Artificial Brownian transport on the nanoscale. Rev. Mod. Phys. 2009, $81,387-442$.

2. Julicher, F.; Ajdari, A.; Prost, J. Modelling molecular motors. Rev. Mod. Phys. 1997, 69, 1269-1281.

3. Marchesoni, F.; Savel'ev, S.; Nori, F. Achieving optimal rectification using underdamped rocked ratchets. Phys. Rev. E 2006, 73, doi:10.1103/PhysRevE.73.021102.

4. Astumian, R.D. Thermodynamics and kinetics of a Brownian motor. Science 1997, 276, 917-922. 
5. Reimann, P. Brownian Motors: Noisy Transport Far from Equilibrium. Phys. Rep. 2002, 361, $57-265$.

6. Jung, J.; Kissner, J.G.; Hanggi, P. Regular and chaotic transport in asymmetric periodic potentials: Inertia ratchets. Phys. Rev. Lett. 1996, 76, 3436-3439.

7. Mateos, J.L. Chaotic transport and current reversal in deterministic ratchets. Phys. Rev. Lett. 2000, 84, 258-261.

8. Son, W.S.; Kim, I.; Park, Y.J.; Kim, C.M. Current reversal with type-I intermittency in deterministic inertia ratchets. Phys. Rev. E 2003, 68, doi:10.1103/PhysRevE.68.067201.

9. Barbi, M.; Salerno, M. Phase locking effect and current reversals in deterministic under-damped ratchets. Phys. Rev. E 2000, 62, 1988-1994.

10. Kenfack, A.; Sweetnam, S.M.; Pattanayak, A.K. Bifurcations and sudden current change in ensembles of classically chaotic ratchets. Phys. Rev. E 2007, 75, doi:10.1103/PhysRevE.75.056215.

11. Mateos, J.L. Current reversals in deterministic chaotic ratchets. Phys. D 2002, 168-169, 205-219.

12. Mateos, J.L. Current reversals in chaotic ratchets: The battle of attractors. Phys. A 2004, 325, 92-100.

13. Mateos, J.L. Intermittency and deterministic diffusion in chaotic ratchets. Commun. Nonlinear Sci. Numer. Simul. 2003, 8, 253-263.

14. Vincent, U.E.; Njah, A.N.; Akinlade, O.; Solarin, A.R.T. Phase synchronization in unidirectionally coupled chaotic ratchets. Chaos 2004, 14, 1018-1025.

15. Vincent, U.E.; Kenfack, A.; Njah, A.N.; Akinlade, O. Bifurcation and chaos in coupled ratchets exhibiting synchronized dynamics. Phys. Rev. E 2005, 72, doi:10.1103/PhysRevE.72.056213.

16. Kostur, M.; Hanggi, P.; Talkner, P.; Meteos, J.L. Anticipated synchronization in coupled inertia ratchets with time-delay feedback. Phys. Rev. E 2005, 72, doi:10.1103/PhysRevE.72.036210.

17. Vincent, U.E.; Laoye, J.A. Synchronization and control of directed trasnport in chaotic ratchets via active control. Phys. Lett. A 2007, 363, 91-95.

18. Guo, L.X.; Hu, M.F.; Xu, Z.Y. Impulsive synchronization and control of directed transport in chaotic ratchets. Chin. Phys. B 2010, 19, doi:10.1088/1674-1056/19/2/020512 .

19. Lu, P.L.; Yang, Y.; Huang, L. Synchronization of linearly coupled networks of deterministic ratchets. Phys. Lett. A 2008, 372, 3978-3985.

20. Zarlenga, D.G.; Larrondo, H.A.; Arizmendi, C.M.; Family, F. Complex synchronization structure of an overdamped ratchet with discontinuous periodic forcing. Phys. Rev. E 2009, 80, doi:10.1103/PhysRevE.80.011127.

21. Xu, S.Y.; Yang, Y.; Song, L. Control-oriented approaches to anticipating synchronization of chaotic deterministic ratchets. Phys. Lett. A 2009, 373, 2226-2236.

22. Sengupta, S.; Guantes, R.; Miret-Artes, S.; Hanggi, P. Controlling directed transport in two-dimensional periodic structures under crossed electric fields. Phys. A 2004, 338, 406-416.

23. Vincent, U.E.; Kenfack, A.; Senthilkumar, D.V.; Mayer, D.; Kurths, J. Current reversals and synchronization in coupled ratchets. Phys. Rev. E 2010, 82, doi:10.1103/PhysRevE.82.046208.

24. Nana-Nbendjo, B.R.; Vincent, U.E.; McClintock, P.V.E. Multi-resonance and enhanced synchronization in stochastically coupled chaotic ratchets. Int. J. Bifurcat. Chaos 2012, 22, doi:10.1142/s0218127412501416. 
25. Vincent, U.E.; Nana-Nbendjo, B.R.; McClintock, P.V.E. Collective dynamics of a network of ratchets coupled via a stochastic dynamical environment. Phys. Rev. E 2013, 87, doi:10.1103/ PhysRevE.87.022913.

26. Borromeo, M.; Costantini, G.; Marchesoni, F. Deterministic ratchets: Route to diffusive transport. Phys. Rev. E 2002, 65, doi:10.1103/PhysRevE.65.041110.

27. Arizmendi, C.M.; Family, F.; Salas-Brito, A.L. Quenched disorder effects on deterministic inertia ratchets. Phys. Rev. E 2001, 63, doi:10.1103/PhysRevE.63.061104.

28. Luchinsky, D.G.; Greenall, M.J.; McClintock, P.V.E. Resonant rectification of fluctuations in a Brownian ratchet. Phys. Lett. A 2000, 273, 316-321.

29. Nayfeh, A.H. Perturbation Methods; Wiley and Sons: New York, NY, USA, 1973.

30. Nayfeh, A.H.; Mook, D.T. Nonlinear Oscillations; Wiley and Sons: New York, NY, USA, 1979.

31. Huberman, B.A.; Cructhfield, J.P.; Packard, N.H. Noise phenomena in Josephson junctions. Appl. Phys. Lett. 1980, 37, 750-752.

32. Lu, P.; Wu, Q.; Yang, Y. Controlling transport and synchronization in non-identical inertial ratchets. J. Optim. Theory Appl. 2013, 157, 888-899.

(C) 2014 by the authors; licensee MDPI, Basel, Switzerland. This article is an open access article distributed under the terms and conditions of the Creative Commons Attribution license (http://creativecommons.org/licenses/by/4.0/). 